\title{
Math-Net.Ru
}

Общероссийский математический портал

В. Е. Подольский, О следе возмущенной операторной полугруппы, Матем. заметки, 2004, том 75, выпуск 3, 462-466

DOI: https://doi.org/10.4213/mzm552 
Использование Общероссийского математического портала Math-Net.Ru подразумевает, что вы прочитали и согласны с пользовательским соглашением http://www. mathnet.ru/rus/agreement

Параметры загрузки:

IP: 34.227 .88 .159

26 апреля 2023 г., 12:45:58

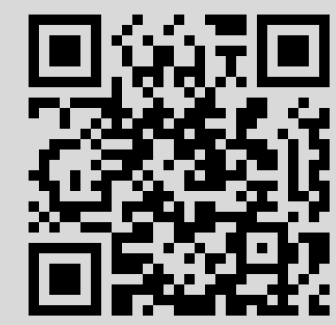




\section{О СЛЕДЕ ВОЗМУЩЕННОЙ ОПЕРАТОРНОЙ ПОЛУГРУППЫ}

\section{В.Е. Подольский}

Работа посвящена изучению следа полугруппы $e^{-t(A+B)}$, где $A$ - дискретный оператор, а $B$ ограниченный оператор, на основе полученньх нами оценок для приближения указанной полугруппы невозмущенной полугруппой $e^{-t A}$ в ядерной норме. Приближения полугрупп в нормах идеалов компактных операторов уже исследовались; мы укажем здесь на работы [1], [2], в которых изучается скорость приближения полугруппы членами последовательности типа Троттера. Однако в основном изучению подвергались конкретные дифференциальные операторы (см., например, [3]), а для абстрактных операторов исследование асимптотики при $t \rightarrow 0+$ следа полугруппы ограничивалось оценкой главной части.

Напомним некоторые факты теории симметрично-нормированных идеалов (в кольце ограниченных операторов) компактных операторов (см. [4]). Синәулярными числами компактного оператора $S$ назьваются упорядоченные по убыванию собственные числа $s_{n}$ положительного оператоpa $\sqrt{S S^{*}}$. Множество компактных операторов со сходяшимся при некотором $p>0$ рядом $\sum s_{n}^{p}$ мы обозначаем $\mathfrak{S}_{p}$ - это множество операторов при $p \geqslant 1$ образует симметрично-нормированньй идеал в алгебре ограниченных операторов с нормой $\|S\|_{p}=\left(\sum s_{n}^{p}\right)^{1 / p}$; симметричность нормы означает выполнение неравенства

$$
\left\|B_{1} S B_{2}\right\|_{p} \leqslant\left\|B_{1}\right\|\|S\|_{p}\left\|B_{2}\right\|
$$

для любых ограниченных операторов $B_{1}$ и $B_{2}$. При $p=1$ эта норма называется ядерной нормой

(C) в. Е. Подольский

2004 
операторов. Так же мы будем использовать неравенство

$$
\|S\|_{p} \leqslant\left\|S_{1}\right\|_{p_{1}} \ldots\left\|S_{k}\right\|_{p_{k}}
$$

здесь $S=S_{1} \ldots S_{k}$, а операторы $S, \ldots, S_{k}$ соответственно принадлежат идеалам $\mathfrak{S}_{p}, \ldots, \mathfrak{S}_{p_{k}}$, где $p^{-1}=p_{1}^{-1}+\cdots+p_{k}^{-1}$.

Во всей работе оператор $A$ таков, что $A^{-1} \in \mathfrak{S}_{p}$ с некоторьм $p \geqslant 1$, его собственные вектоpa $\left\{\varphi_{n}\right\}_{n=1}^{\infty}$ образуют ортонормированный базис, соответствующие собственные числа $\left\{\lambda_{n}\right\}_{n=1}^{\infty}$ лежат в открытом секторе $\Omega:-\pi / 2+\omega<\arg z<\pi / 2-\omega, 0<\omega<\pi / 2$. Нетрудно видеть, что тогда $A^{*}$ имеет те же собственные вектора с собственными числами $\bar{\lambda}_{n}$ и $A A^{*}=A^{*} A$. Через $|A|$ мы будем обозначать положительный оператор $\sqrt{A A^{*}}$; его дробные степени определены по спектральной теореме. Так как спектр $A$ лежит в $\Omega$, то $\left|\lambda_{n}\right|^{-1} \geqslant\left(\operatorname{Re} \lambda_{n}\right)^{-1} \cos \omega$ и $(\operatorname{Re} A)^{-1} \in \mathfrak{S}_{p}$, и так как сингулярные числа полугруппы $e^{-t A}$ равны $e^{-\operatorname{Re} \lambda_{n} t}$, верна формула

$$
\left\|e^{-t A}\right\|_{p}=\left(\sum_{n=1}^{\infty} e^{-p \operatorname{Re} \lambda_{n} t}\right)^{1 / p}
$$

Об операторе $B$ мы предполагаем, помимо ограниченности, что спектр $A+B$ так же лежит в $\Omega$ (техническое удобство, не ограничивающее общности). Через $\mu_{n}$ будем обозначать собственные числа оператора $A+B$. Во всей работе одним и тем же символом $c$ обозначаются различные положительные константы, не зависящие от основных переменных встречающихся неравенств.

Основным соотношением, которое будет использовано для исследования возмущенной полугруппы, будет следующее хорошо известное уравнение [5]:

$$
e^{-t(A+B)}-e^{-t A}=-\int_{0}^{t} e^{-(t-s) A} B e^{-s(A+B)} d s
$$

из которого методом последовательных приближений получаем сходящийся по операторной норме ряд $\left(t=t_{0}\right)$

$$
e^{-t(A+B)}=e^{-t A}+\sum_{k=1}^{+\infty}(-1)^{k} \int_{0}^{t} \int_{0}^{t_{1}} \ldots \int_{0}^{t_{k-1}} e^{-\left(t-t_{1}\right) A} B \ldots B e^{-t_{k} A} d t_{k} \ldots d t_{1} .
$$

Tеорема 1. Пусть существует число $\alpha>0$ maкое, что $\left\|e^{-t A}\right\|_{1} \leqslant c t^{-\alpha}$ nрu $t \in(0$, $+\infty)$. Тогда ряд (5) сходится в ядерной норме, причем сходимость остатка ряда равномерна на любом отрезке $[a, b] \in[0,+\infty)$.

ДоКАЗАТЕЛЬСтво. Используя неравенства (1) и (2), запишем оценку общего члена ряда (5) (как мы увидим ниже, преобразования законны для всех достаточно больших $k$ ):

$$
\begin{aligned}
& \left\|\int_{0}^{t} \int_{0}^{t_{1}} \ldots \int_{0}^{t_{k-1}} e^{-\left(t-t_{1}\right) A} B e^{-\left(t_{1}-t_{2}\right) A} \ldots B e^{-t_{k} A} d t_{k} \ldots d t_{1}\right\|_{1} \\
& \quad \leqslant\|B\|^{k} \int_{0}^{t} \int_{0}^{t_{1}} \cdots \int_{0}^{t_{k-1}}\left\|e^{-\left(t-t_{1}\right) A}\right\|_{k+1}\left\|e^{-\left(t_{1}-t_{2}\right) A}\right\|_{k+1} \ldots\left\|e^{-t_{k} A}\right\|_{k+1} d t_{k} \ldots d t_{1} .
\end{aligned}
$$

Так как из (3) следует $\left\|e^{-t A}\right\|_{k}=\left\|e^{-k t A}\right\|_{1}^{1 / k} \leqslant c(k t)^{-\alpha / k} \leqslant c t^{-\alpha / k}$, интеграл в (6) оценивается сходящимся при $k$ таких, что $\alpha /(k+1)<1$, интегралом

$$
\int_{0}^{t} \int_{0}^{t_{1}} \ldots \int_{0}^{t_{k-1}}\left(t-t_{1}\right)^{-\frac{\alpha}{k+1}}\left(t_{1}-t_{2}\right)^{-\frac{\alpha}{k+1}} \ldots t_{k}^{-\frac{\alpha}{k+1}} d t_{k} \ldots d t_{1}=\frac{\Gamma^{k+1}\left(1-\frac{\alpha}{k+1}\right)}{\Gamma(k+1-\alpha)} t^{k-\alpha} .
$$

Мы вычислили этот интеграл, последовательно интегрируя по $t_{k}, \ldots, t_{1}$ и пользуясь Эйлеровым интегралом $\int_{0}^{\tau} t^{\gamma}(\tau-t)^{\beta} d t=\tau^{\gamma+\beta+1} B(\gamma+1, \beta+1)$ при $\beta, \gamma>-1$. Так как $\Gamma(k)$ с точностью до экспоненциальных множителей ведет себя как $k^{k}$, и так как все остальные коэффициенты в нашей оценке $^{1}$ общего члена ряда (5) имеют не более чем экспоненциальный рост, теорема доказана.

\footnotetext{
${ }^{1} \mathrm{O}$ константе в формуле (7) заметим, что $\lim _{k \rightarrow \infty} \Gamma^{k+1}\left(1-\frac{\alpha}{k+1}\right)=e^{\gamma \alpha}$, здесь $\gamma$ - постоянная Эйлера.
} 
СлЕДСтвиЕ 1. В условиях теоремы 1 при всех $N \in \mathbb{N}$ таких, что $\alpha / N<1$, верна асимптотическая при $t \rightarrow 0+$ формула для следа возмущенной полугруппь

$$
\begin{aligned}
& \operatorname{Tr} e^{-t(A+B)}=\operatorname{Tr} e^{-t A}-\operatorname{Tr}\left(B e^{-t A}\right) t \\
&+\sum_{k=2}^{N-2}(-1)^{k} \operatorname{Tr} \int_{0}^{t} \cdots \int_{0}^{t_{k-1}} e^{-\left(t-t_{1}\right) A} B \ldots B e^{-t_{k} A} d t_{k} \ldots d t_{1}+O\left(t^{N-1-\alpha}\right) .
\end{aligned}
$$

ДоКАЗАТЕЛЬСтво. Оценка остатка немедленно следует из доказательства теоремы, второе слагаемое имеет приведенный вид в силу известного свойства следа $\operatorname{Tr} A B=\operatorname{Tr} B A$ :

$$
\int_{0}^{t} \operatorname{Tr}\left(e^{-(t-s) A} B e^{-s A}\right) d s=\int_{0}^{t} \operatorname{Tr}\left(B e^{-t A}\right) d s .
$$

СлЕДСТВИЕ 2. Если вместо условия теоремы 1 при $t \rightarrow 0+$ верна асимптотическая оченка $\left\|e^{-t A}\right\|_{1}=o\left(t^{-\alpha}\right)$, то в формуле (8) (по-прежснему верной при тех же $N$ ) остаточный член имеет вид $о\left(t^{N-1-\alpha}\right)$.

ДоКАЗАТЕльСТво. При этом условии для любого $\varepsilon>0$ в достаточно малой правой полуокрестности точки $t=0$ в подынтегральной функции интеграла $(7)$ вместо сомножителя $t_{k}^{-\alpha /(k+1)}$ можно взять $\varepsilon t_{k}^{-\alpha /(k+1)}$.

ЗАмеч АниЕ 1 . Доказанная теорема 1 (и соответственно следствия 1 и 2 ) никак не использовали структуру спектра оператора и полученные результаты имеют место для операторов с произвольной природой спектра. Однако на практике удобно пользоваться известными свойствами спектра; здесь мы укажем хорошо известные достаточные условия на спектр дискретного оператора с тем, чтобы к нему были применимы теорема и следствия:

i) если $\lambda_{n} \in \Omega$ и $\left|\lambda_{n}\right| \geqslant c n^{1 / \alpha}$ с некоторым $\alpha>0$, то $\left\|e^{-t A_{1}}\right\|_{1} \leqslant c t^{-\alpha}$;

ii) если $\lambda_{n} \in \Omega$ и $\left|\lambda_{n}^{-1}\right|=o\left(n^{-1 / \alpha}\right)$ с некоторым $\alpha>0$, то $\left\|e^{-t A}\right\|_{1}=o\left(t^{-\alpha}\right)$ при $t \rightarrow 0+$.

Далее мы применим полученные результаты к теории регуляризованных следов. В работе [6] для оператора $A$ с ядерной резольвентой и ограниченного $B$ была доказана формула следа, просуммированного по Абелю:

$$
\lim _{t \rightarrow 0+} \sum_{n=1}^{\infty}\left(\mu_{n}-\lambda_{n}-\left(B \varphi_{n}, \varphi_{n}\right)\right) e^{-\lambda_{n} t}=0 .
$$

Следующая теорема усиливает этот результат.

Теорема 2. Если $\left|\lambda_{n}\right|^{-1}=o\left(n^{-1}\right)$, то формула (9) верна.

ДокАЗАТЕльство. Как указьвалось выше, в данном случае имеет место оценка

$$
\left\|e^{-t A}\right\|_{1}=o\left(t^{-1}\right)
$$

при $t \rightarrow 0+$, и в силу следствия 2 верна формула для следа возмущенной полугруппы (здесь мы положили $N=3$ )

$$
\operatorname{Tr} e^{-t(A+B)}=\operatorname{Tr} e^{-t A}-t \operatorname{Tr}\left(B e^{-t A}\right)+o(t) .
$$

Используя несложное преобразование

$$
\frac{1}{t}\left(\operatorname{Tr} e^{-t(A+B)}-\operatorname{Tr} e^{-t A}\right)=\frac{1}{t} \sum_{n=1}^{\infty}\left(e^{-\mu_{n} t}-e^{-\lambda_{n} t}\right)=\frac{1}{t} \sum_{n=1}^{\infty}\left(e^{\left(\lambda_{n}-\mu_{n}\right) t}-1\right) e^{-\lambda_{n} t}
$$


и ограниченность $B$ (для всех $n$ верно $\left|\mu_{n}-\lambda_{n}\right|<c$ ), мы можем в окрестности нуля записать

$$
\operatorname{Tr}\left(B e^{-t A}\right)+o(1)=\sum_{n=1}^{\infty}\left(\mu_{n}-\lambda_{n}\right) e^{-\lambda_{n} t}+O(t) \sum_{n=1}^{\infty} e^{-\lambda_{n} t}
$$

и далее, вычисляя $\operatorname{Tr}\left(B e^{-t A}\right)$ в базисе $\left\{\varphi_{n}\right\}$ и используя $\sum_{n=1}^{\infty} e^{-\lambda_{n} t}=o\left(t^{-1}\right)$, получаем

$$
\sum_{n=1}^{\infty}\left(B \varphi_{n}, \varphi_{n}\right) e^{-\lambda_{n} t}=\sum_{n=1}^{\infty}\left(\mu_{n}-\lambda_{n}\right) e^{-\lambda_{n} t}+o(1),
$$

что и доказывает теорему.

В конкретньх задачах математической физики вычисление слагаемых в формуле (8) при $k \geqslant 2$ уже сопряжено с заметньпи техническими трудностями (при $k=2$ для псевдодифференциального оператора $A$ на компактном многообразии размерности $m$, возмущенного оператором умножения на функцию, речь идет о вычислении $(4 m+2)$-кратного интеграла). В следующей теоремемы предлагаем упрощение этих формул при некоторых дополнительных предположениях об операторах, сравнительно легко проверяемых для псевдодифференциальных операторов. Мы ограничиваемся наиболее важньм (и наименее громоздким) случаем $k=2$. Повторение этих рассуждений для следуюших членов разложения не содержит никаких иных трудностей, помимо объемности выкладок.

Теорема 3. Пусть условия теоремы 1 выполнены при $\alpha<4$ и пусть существует $-\alpha<\delta<1-\alpha / 4$ mакое, что оператор $(B A-A B)|A|^{-\delta}$ продолхсается до ограниченного. Тогда при $t \rightarrow 0+$ верно

$$
\operatorname{Tr} e^{-t(A+B)}=\operatorname{Tr} e^{-t A}-t \operatorname{Tr}\left(B e^{-t A}\right)+\frac{t^{2}}{2} \operatorname{Tr}\left(B^{2} e^{-t A}\right)+O\left(t^{3-\alpha-\delta}\right) .
$$

ДоКАЗАТЕЛЬСтво. При $\alpha<4$ формула (8) приобретает вид $(N=4)$

$$
\begin{aligned}
\operatorname{Tr} e^{-t(A+B)}= & \operatorname{Tr} e^{-t A}-\operatorname{Tr}\left(B e^{-t A}\right) t \\
& +\operatorname{Tr} \int_{0}^{t} \int_{0}^{s} e^{-(t-s) A} B e^{-(s-\tau) A} B e^{-\tau A} d \tau d s+O\left(t^{3-\alpha}\right)
\end{aligned}
$$

и в дополнительном изучении нуждается только третье слагаемое. Воспользуемся формулой $[7$, приложение 1]

$$
B e^{-t A}-e^{-t A} B=t \int_{0}^{1} e^{-(1-\xi) t A}(A B-B A) e^{-\xi t A} d \xi
$$

Тогда

$$
\begin{aligned}
& \int_{0}^{t} \int_{0}^{s} e^{-(t-s) A} B e^{-(s-\tau) A} B e^{-\tau A} d \tau d s=\int_{0}^{t} \int_{0}^{s} e^{-(t-s) A} B\left(B e^{-(s-\tau) A}\right. \\
& \left.\quad+(s-\tau) \int_{0}^{1} e^{-(1-\xi)(s-\tau) A}(B A-A B) e^{-\xi(s-\tau) A} d \xi\right) e^{-\tau A} d \tau d s \\
& =\int_{0}^{t} \int_{0}^{s} e^{-(t-s) A} B^{2} e^{-s A} d \tau d s \\
& \quad+\int_{0}^{t} \int_{0}^{s} e^{-(t-s) A} B(s-\tau) \int_{0}^{1} e^{-(1-\xi)(s-\tau) A}(B A-A B) e^{-\xi(s-\tau) A} d \xi \cdot e^{-\tau A} d \tau d s .
\end{aligned}
$$

Относительно первого слагаемого замечаем, что

$$
\operatorname{Tr} \int_{0}^{t} \int_{0}^{s} e^{-(t-s) A} B^{2} e^{-s A} d \tau d s=\int_{0}^{t} \int_{0}^{s} \operatorname{Tr}\left(B^{2} e^{-t A}\right) d \tau d s=\frac{t^{2}}{2} \operatorname{Tr}\left(B^{2} e^{-t A}\right)
$$


и для доказательства теоремы нам осталось оценить второе слагаемое. Так как оператор ( $B A$ $-A B)|A|^{-\delta}$ продолжается до ограниченного, то

$$
\begin{aligned}
& \left\|\int_{0}^{t} \int_{0}^{s} e^{-(t-s) A} B(s-\tau) \int_{0}^{1} e^{-(1-\xi)(s-\tau) A}(B A-A B) e^{-\xi(s-\tau) A} e^{-\tau A} d \xi d \tau d s\right\|_{1} \\
& \quad \leqslant c \int_{0}^{t} \int_{0}^{s}(s-\tau) \int_{0}^{1}\left\|e^{-(t-s) A}\right\|_{4}\left\|e^{-(1-\xi)(s-\tau) A}\right\|_{4}\left\|e^{-\xi(s-\tau) A}\right\|_{4}\left\|\left.A\right|^{\delta} e^{-\tau A}\right\|_{4} d \xi d \tau d s
\end{aligned}
$$

В работе [8] проведено детальное исследование поведения в окрестности нуля функции вида $\sum \lambda_{n}^{\delta} e^{-\lambda_{n} t}$ по известному поведению функции $\sum e^{-\lambda_{n} t}$ и из этих результатов следует, что ${ }^{2}$

$$
\left\||A|^{\delta} e^{-t A}\right\|_{4} \leqslant c t^{-\alpha / 4-\delta}
$$

Применяя эту оценку и уже имеющиеся оценки норм экспонент, получаем вычисляемьй интеграл

$$
\int_{0}^{t} \int_{0}^{s} \int_{0}^{1}(t-s)^{-\alpha / 4}(1-\xi)^{-\alpha / 4}(s-\tau)^{1-\alpha / 2} \xi^{-\alpha / 4} \tau^{-\alpha / 4-\delta} d \xi d \tau d s=c t^{3-\alpha-\delta}
$$

и оценка остаточного члена доказана.

В качестве примера возможного применения теоремы 3 укажем на задачу вычисления следа экспоненты оператора Лапласа-Бельтрами с потенциалом на компактном многообразии. В этом случае $\delta=1 / 2$, и наша теорема позволяет для решения этой задачи при размерности многообразия $m \leqslant 7$ просто воспользоваться готовыми формулами работы [9] для выражений вида $\operatorname{Tr}\left(f e^{t \Delta}\right)$, где $f$ - гладкая функция, а $-\Delta$ - оператор Лапласа на многообразиях без края или оператор одной из классических краевых задач. При размерности $m=2$ остаточный член есть $O\left(t^{3 / 2}\right)$.

\section{СПИСОК ЦИТИРОВАННОЙ ЛИТЕРАТУРЫ}

1. Ichinose T., Tamura H. // Asymptot. Anal. 1998. V. 17. P. 239-266. 2. Takanobu S. // Osaka J. Math. 1998. V. 35. № 3. P. 659-682. 3. Simon B. // Bull. AMS. 1982. V. 7. № 3. P. 447-526. 4. Гохберг И. Ц., Крейн М. Г. Введение в теорию линейных несамосопряженных операторов в гильбертовом пространстве. М.: Наука, 1965. 5. Като Т. Теория возмущений линейных операторов. М.: Мир, 1972. 6. Подольский В. Е. // Вестн. МГУ. Сер. 1. 1999. № 5. С. 42-48. 7. Карасев М. В., Маслов В. П. Нелинейные скобки Пуассона. Геометрия и квантование. М.: Наука, 1991. 8. Подольский В. Е. // Матем. заметки. 1994. Т. 56. № 1. С. 71-77. 9. Gilkey P. В., Branson T. P. // Comm. Partial Differential Equations. 1990. V. 15. № 2. P. 245-272.

Московский государственный университет им. М.В. Ломоносова

Поступило 30.01 .2003

\section{К ВОПРОСУ О ВОЗМУЩЕНИЯХ СИСТЕМЫ ХААРА}

\section{П. А. Терехин}

В статье обсуждаются свойства полноты и базисности в пространстве $L_{p}[0,1], 1 \leqslant p<\infty$, общих систем функций

$$
\left\{1, \varphi_{k, j}\right\}, \quad k=0,1, \ldots, \quad j=0, \ldots, 2^{k}-1
$$

\footnotetext{
${ }^{2}$ Здесь используется условие $\delta>-\alpha . \mathrm{B}[8]$ исследованы все случаи, но для наших целей этот
} представляет наибольший интерес. 